This item was submitted to Loughborough's Research Repository by the author.

Items in Figshare are protected by copyright, with all rights reserved, unless otherwise indicated.

\title{
Intermittent and metastable chaos in a memristive artificial neuron with inertia
}

PLEASE CITE THE PUBLISHED VERSION

https://doi.org/10.1016/j.chaos.2020.110383

PUBLISHER

Elsevier BV

VERSION

AM (Accepted Manuscript)

\section{PUBLISHER STATEMENT}

This paper was accepted for publication in the journal Chaos, Solitons \& Fractals and the definitive published version is available at https://doi.org/10.1016/j.chaos.2020.110383.

LICENCE

CC BY-NC-ND 4.0

\section{REPOSITORY RECORD}

Wojtusiak, AM, Alexander Balanov, and Sergey Saveliev. 2020. "Intermittent and Metastable Chaos in a Memristive Artificial Neuron with Inertia”. Loughborough University. https://hdl.handle.net/2134/13174496.v1. 


\title{
Intermittent and metastable chaos in a memristive artificial neuron with inertia.
}

\author{
A. M. Wojtusiak, A. G. Balanov and S. E. Savel'ev \\ Department of Physics, Loughborough University, Loughborough LE11 3TU, UK
}

\begin{abstract}
Inspired by rapid experimental development of diffusive memristors, we propose a computational model of a memristive artificial neuron that takes into consideration inertia of metallic nanoparticles within the dielectric layer of the core-memristor. This model displays rich nonlinear dynamics, which has been speculated to be key for successful emulation of living biological neurons by neuromorphic devices. We found out four characteristic dynamical regimes realized in the system depending on inertness of the nanoparticles. For low-inertia particles, the artificial neuron biased by an applied DC-voltage demonstrates either steady state or regular periodic oscillations. For higher inertia, metastable and intermittent chaos can appear in the system. We analyse the transitions between these regimes and draw parallels between our model and biological neurons.
\end{abstract}

Keywords: intermittent chaos, metastable chaos, memristor, artificial neuron

\section{Introduction}

Artificial intelligence (AI) is now a commonplace in the modern world. It has a rich variety of important applications, such as generation and analysis of big data $[1,2]$ or image recognition $[3,4]$, and has great potential for applications in medicine, e.g. for cancer diagnosis $[5,6]$. However, the current state-of-the-art machine learning technologies utilise conventional CPU- and GPU-based computers. This requires a much larger amount of power and time to reach the results comparable to what the human brain can achieve almost instantly, with little power used. Therefore, nowadays research is carried out into alternative approaches of machine learning, referred to as neuromorphic computing, which closer resemble the behaviours of biological neuron networks. One of the promising directions here is the use of a network of memristors, as they have been shown to have properties similar to biological neurons, and can be used as building elements in neuromorphic computing $[7,8,9]$.

The memristor was theoretically proposed by Leon Chua [10], and for the first time experimentally realized in HP Labs [11]. This device changes its resistance depending on the electric current or voltage prehistory, e.g. on the total charge flown through it. Such a feature makes the memristor one of the most promising elements for hardware realisation of AI mimicking biological neurons [12]. To date a variety of different types of memristors have been proposed and developed that utilize different physical principles and charge transport mechanisms for realization of memristive function, see reviews in [7] and [13].

Recently, a new type of a memristor has been fabricated, measured and modelled [14]. This is a two-terminal solid-state device with a dielectric thin film sandwiched between two inert metal electrodes. Ag-nanoparticles are dissolved within the thin film and are able to diffuse between the electrodes.

If a bias voltage is applied to the electrodes of such a memristor being initially in a high-resistance state, the metal nanoparticles diffuse throughout the dielectric layer and eventually coalesce. Such coalescence forms a conductive bridge, or filament, which switches the device to a low resistive state. When the voltage decreases, the filament breaks apart and the memristor returns to its high resistive state. It has been shown that in such devices, called "diffusion memristors", the mechanical, electric and 
heat degrees of freedom intricately interplay, making their dynamics very rich and fascinating $[14,15]$.

A network of artificial neurons consisting of diffusive and standard non-volatile memristors has recently demonstrated its high efficiency in image recognition [16]. However, most of the artificial neuron networks developed so far use training to adjust resistive connection between links and use artificial neurons as a readout device. This is very different from the way our brain operates. Indeed, information in the brain is transferred via electrical signals in the form of current (charge) spiking generated by very complex networks of neuron cells. Because of its plasticity and constant selfadaptation to information coming from sensory system it is widely assumed that the brain lives "on the edge of chaos", i.e. at a critical transition point between randomness and regularity [17]. Furthermore, studies have shown that some processes that form the basis for consciousness relate to metastability - the way in which signals in the brain remain outside their equilibrium or dynamical attractors for a prolonged amount of time as they interact or interfere with one another $[18,19]$. Conventional computational models of artificial neural networks usually lack or ignore this behaviour, which nonetheless can be key in replicating biological neural activity. Moreover, the decades of previous research clearly demonstrated that complex stochastic and chaotic dynamics are quite common in a broad range of physical systems including mechanical falling discs [20], nonlinear circuits [21, 22, 23], superconducting vortices [24], nanoparticle transport [25], optomechanical devices [26, 27], to name a few. Dynamical regimes and mechanisms of their emergence can be very different from ones typical of regular behaviour, and therefore require additional thorough study.

Previously, it has been experimentally shown that memristor circuits are indeed able to demonstrate chaotic behaviour [28, 29]. However, in order to describe the transitions to chaos, periodic temperature oscillations were introduced in the corresponding model [28]. On the other hand, some stochastic models of diffusive memristors [14] have transitions from highly coherent to very erratic spiking behaviour, however there is yet no clear understanding whether the observed irregularity has deterministic or stochastic origin.

Here we propose a deterministic model inspired by the stochastic dynamics of a diffusive memristor. Namely, we consider underdamped mechanical mo- tion of an Ag-cluster in a gap between two arms of a conducting bridge driven by both an electric field and a temperature gradient inside a single well potential. We reveal dynamical regimes appearing in the system with variation of inertness of the Agnanoparticles and analyse the transitions between them.

\section{Model}

The resistance of the considered here memristor (or memristance) $R_{m}$ is a function of the state variable, $x$, which in our case represents the positions of metallic nanoparticles between the two conductive terminals, such that when $x=0$, the particle is equidistant to both. The inertness of the particle is characterised by mass parameter $m$. Note that the origin of the inertia can be linked either directly to bare mass of the Ag-nanoparticle or arise from the slow relaxation dynamics of the insulating matrix where the Ag-cluster diffuses. Indeed, Agnanoparticles elastically deform the surrounding insulating matrix and these elastic deformations can relax, generating an additional time-scale, which is equivalent to the effective mass of an Ag-cluster.

Ordinary differential equations (ODEs) were used to describe the underdamped dynamics of the Ag-nanocluster. They can be derived by considering forces acting upon the particle when the biasvoltage $V_{m}$ is applied to the memristor:

$$
\frac{d x}{d t}=y
$$

$$
m \frac{d y}{d t}=\overbrace{-k y}^{\begin{array}{c}
\text { friction } \\
\text { force }
\end{array}}-\underbrace{\frac{d U}{d x}}_{\begin{array}{c}
\text { potential } \\
\text { gradient }
\end{array}}+\overbrace{q_{1} V_{m}}^{\begin{array}{c}
\text { electric } \\
\text { force }
\end{array}}-\underbrace{q_{2} f}_{\begin{array}{c}
\text { thermal } \\
\text { force }
\end{array}}
$$

where $t$ is time and the potential force

$$
-\frac{d U}{d x}=(x-a)^{2}+a+b e^{-\left(\frac{x-c}{a}\right)^{2}}+8 d x^{7} e^{d x^{8}}
$$

with parameters $a, b, c, d$, corresponds to an asymmetric one-well potential which takes into account nanoparticle-pinning energy [14] as well as interfacial energy responsible for formation of large metallic clusters near one of the terminals in an asymmetric device [30]. Pinning originates due to interactions of Ag-nanoparticles with impurities, the substrate and/or the ionic lattice $[31,32,33]$. Equation (1a) defines the nanoparticle velocity $y=d x / d t$, 
while (1b) determines acceleration by forces acting on the particle: friction, potential gradient, electric force (with induced nanoparticle charge $q_{1}$ ), and thermal gradient force. The model takes into account thermophoretic and/or thermoelectric effects causing an additional "thermal" force proportional to the temperature gradient $f[34,35]$ (with effective "thermal charge" $q_{2}$ ).

In our calculations we use the following dimensionless parameters: $k=1, q_{1}=0.63, q_{2}=1.1, a=$ $0.1, b=80, c=0.8$ and $d=1.3$. Note that the general behaviour and transition between regimes discussed below are robust to variation of this parameter set, but the regions on the parameter plane $\left(V_{m}, m\right)$ corresponding to different regimes depend on the particular choice of the parameters.

An ODE describing the dynamics of the temperature gradient $f$ was obtained by considering the rate of change of temperature $T$ (Newton's cooling law):

$$
\begin{aligned}
\frac{d T}{d t} & =\frac{Q}{C_{T}}-\kappa\left(T-T_{0}\right) \\
& =\frac{V_{m}^{2}}{C_{T} R_{m}}-\kappa\left(T-T_{0}\right)
\end{aligned}
$$

where $Q=V_{m}^{2} / R_{m}$ is the heating power. It was earlier shown that the cooling law (2) can produce the current-controlled negative differential resistance in memristors [36]. Below we use the heat capacity $C_{T}=0.12$, and the heat transfer coefficient $\kappa=0.6$, while the ambient temperature $T_{0}$ does not affect the considered here thermal force. Now we need to evaluate the temperature gradient. In order to do this we assume that the particle deviates from its trajectory $x(t)$, such as $x=x(t)+\delta x$. It results in deviation of the corresponding temperature and voltage: $T=T(t)+f \delta x$ and $V_{m}=V_{m}(t)+\mathcal{V} \delta x$. Substituting all these expression in (2), expanding $x$-dependent expressions up to the first order, and eliminating $\delta x$ gives:

$$
\frac{d f}{d t}=\frac{2 V_{m} \mathcal{V}}{C_{T} R_{m}}-\frac{V_{m}^{2} R_{m}^{\prime}}{C_{T} R_{m}^{2}}+\kappa f
$$

where symbol' refers to $x$-derivatives of $R_{m}$.

The artificial neuron can be represented by a simple circuit consisting of the memristor of varying resistance $R_{m}$, a capacitor $C$ and a load resistor $R_{1}$ (for simulations we used $R_{1}=100$ ), as shown in Fig. 1. Using Kirchhoff's laws, the rate of change

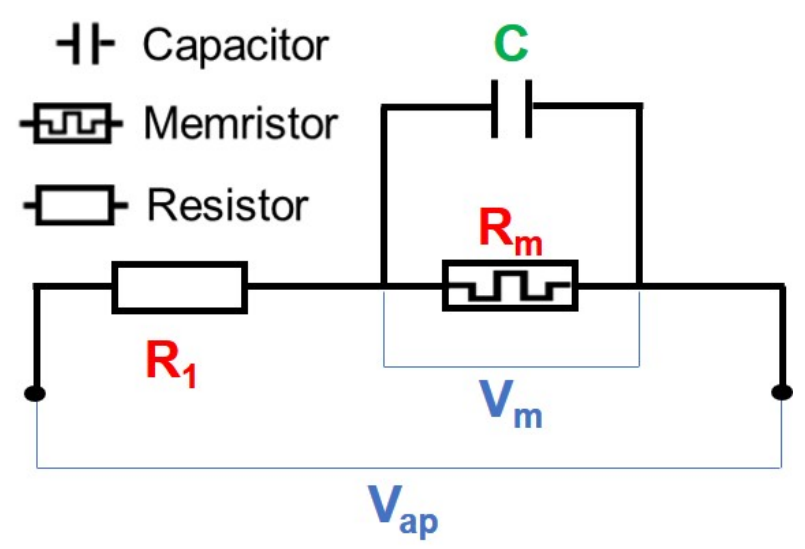

Figure 1: A circuit diagram of the model used in this paper. The memristor $R_{m}$ is in parallel with a capacitor $C$ and in series with a load resistor $R_{1} ; V_{a p}$ is the applied bias $D C$ voltage and $V_{m}$ is potential difference across the memristor.

of $V_{m}$ can be described as:

$$
\tau_{0} \frac{d V_{m}}{d t}=V_{a p}-\left(1+\frac{R_{1}}{R_{m}(x)}\right) V_{m}
$$

where $\tau_{0}=R_{1} C$ is the relaxation time constant and $V_{a p}$ is the applied voltage.

An ODE describing $\mathcal{V}$ has been obtained by using Equation (4) in a similar maner as for deriving equation for $f$ :

$$
\tau_{0} \frac{d \mathcal{V}}{d t}=-\left(1+\frac{R_{1}}{R_{m}(x)}\right) \mathcal{V}+\frac{R_{1} R_{m}^{\prime}(x)}{R_{m}^{2}} V_{m}
$$

To complete the model, we assume electron tunneling between the device terminals and the Agcluster with the rate exponentially decreasing with the tunneling length $\lambda$. This implies the tunneling resistance $R_{m}(x)$ depending on Ag-nanoparticle position [15]:

$$
\begin{aligned}
& R_{m}=R_{0} \cosh \left(\frac{x}{\lambda}\right) \\
& R_{m}^{\prime}=\frac{R_{0}}{\lambda} \sinh \left(\frac{x}{\lambda}\right)
\end{aligned}
$$

with the minimal resistance $R_{0}$ corresponding to the low-resistance state. We use $R_{0}=1$ and $\lambda=$ 0.12 in our simulations.

To solve the ODEs described by Equations (1)(6), the second order Runge-Kutta method was implemented, with a time step size of $10^{-5}$. This method and step size were chosen as they provided high levels of accuracy, while remaining simple enough to achieve results in reasonable timescales. The local error for each step is on the order of $10^{-15}$ and the global error on the order of $10^{-10}$. 


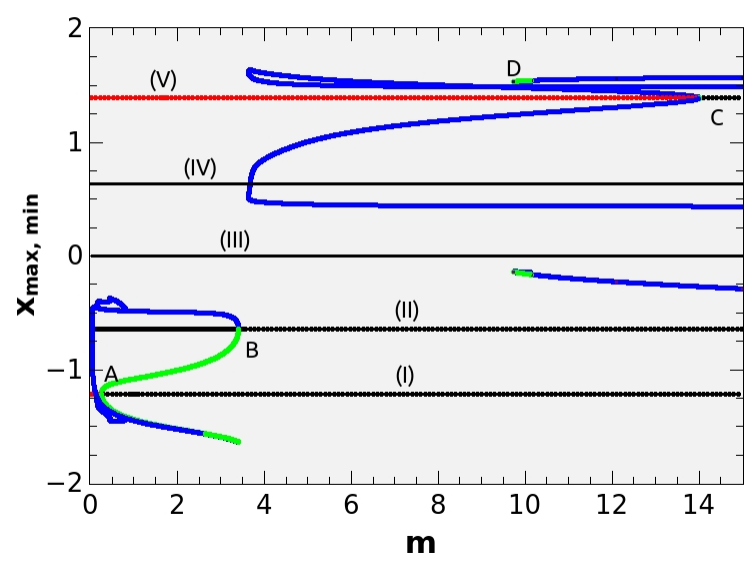

Figure 2: Bifurcation diagram showing maxima $x_{\max }$ and minima $x_{\text {min }}$ of the particle position $x(t)$ and the stability of the dynamical regimes for a range of mass values $m$ : green corresponds to a stable limit cycle, blue - unstable limit cycle, red - stable fixed points, black - unstable fixed points; $(I)-(V)$ denotes the positions of specific fixed points; $A-D$ highlight the bifurcations discussed in the text.

\section{Results and Discussion}

To study the effects of inertia on the dynamics of our model, we fix the applied voltage $V_{a p}=70$, and analyse how solutions of the model (1)-(6) change for different values of $m$. Fig. 2 displays a bifurcation diagram that summarises the behaviour of $x$ for $0<m<15$. The figure shows the evolution and stability of regular (equilibrium and periodic) solutions of the model as $m$ changes. The points indicate the maximal $x_{\max }$ and minimal $x_{\min }$ values of stationary solutions of $x(t)$ for the given value of $m$. Obviously, $x_{\max }$ and $x_{\min }$ coincide for the equilibrium (steady state) solutions, and designate the span of the oscillations for the periodic solutions. The red and black points correspond to stable and unstable steady states, respectively. Green points indicate the stable periodic solutions, while blue points represent the unstable limit cycles. As the figure shows, the system has five equilibrium points labelled as (I)-(V). Their mutual allocation affect the topology of the phase trajectories, and therefore predefine the character of the observed oscillations (see e.g. Fig. 4). The fixed points labelled (I) and $(\mathrm{V})$ in Fig. 2 change their stability with variation of $m$ at the points $A$ and $C$, respectively, thus giving birth to the stable (green) and unstable (blue) limit cycles. Another stable limit cycle appears at point

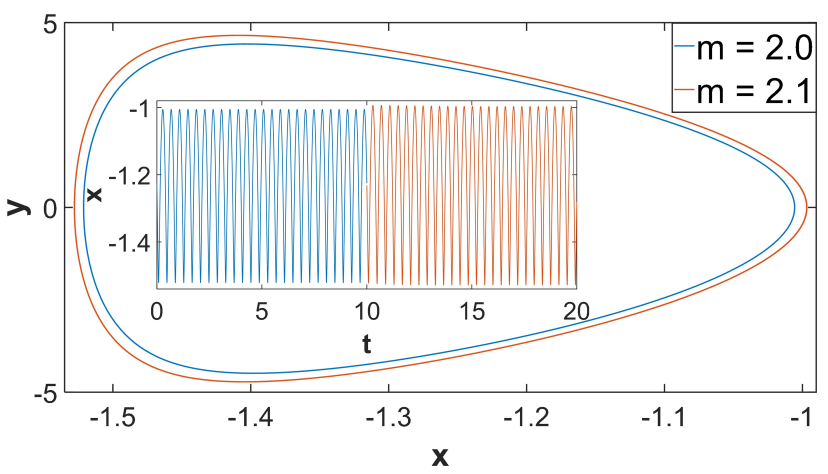

Figure 3: The projection of the phase trajectories corresponding to stationary periodic oscillations to the plane $(x, y)$, for $m=2$ (blue) and $m=2.1$ (red). The inset shows the respective time plot $x(t)$ for $m=2$ (blue) and $m=2.1$ (red); to simplify comparison the plot for $m=2.1$ is offset by time interval $\Delta t=10$.

$D$ in the result of saddle-node bifurcation for limit cycles. In their turn, the limit cycles bifurcate further producing new limit sets in the phase space. Accumulation of various limit sets with different stability creates the conditions for appearance of both long living metastable oscillations and chaos. For $m>3.4$, there are metastable chaotic oscillations that last for a finite amount of time, after which they converge to a stable fixed point. This metastable chaos exists up to $m \approx 14$ (see Section 3.1 ). For $m>14$, the fixed point becomes unstable and the system enters its chaotic regime (see Section 3.2). The system also has a small region of periodicity around $m=10$, where a limit cycle in the positive $x$-values is reached.

\subsection{Metastability}

As Fig. 2 shows, with increase of $m$ the fixed point (I) near the bottom electrode losses its stability at $m \approx 0.26$ (point $A$ ) via the AndronovHopf bifurcation thus causing appearance of stable periodic oscillations, whose $x_{\min }$ and $x_{\max }$ are represented by green points. With increasing $m$, the oscillation amplitude $\left(x_{\max }-x_{\min }\right) / 2$ grows. Fig. 3 illustrates this amplitude change by presenting the projection of the phase trajectories onto the phase plane $(x, y)$ and the respective time plot of stationary $x(t)$ shown in the inset for $m=2.0$ (blue curve) and $m=2.1$ (red curve). At $m \approx 3.409$ the corresponding limit cycle collides with an unstable fixed point (II) at point $B$. After this collision the periodic oscillations disappear giving rise to longlasting irregular oscillations which finally converge 


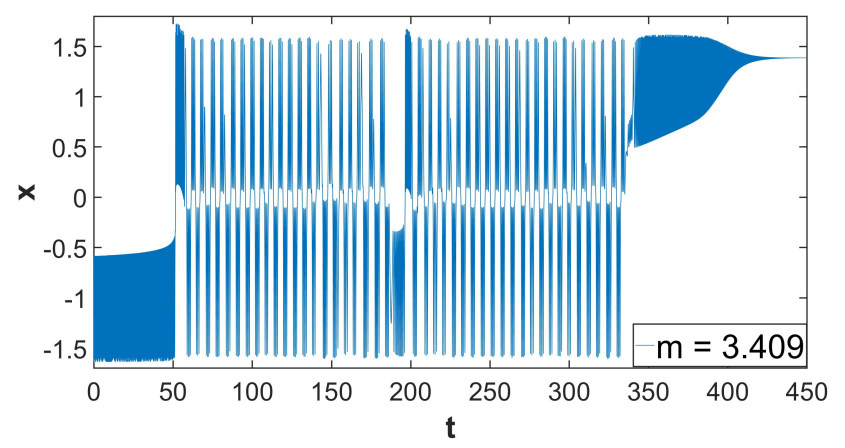

(a)

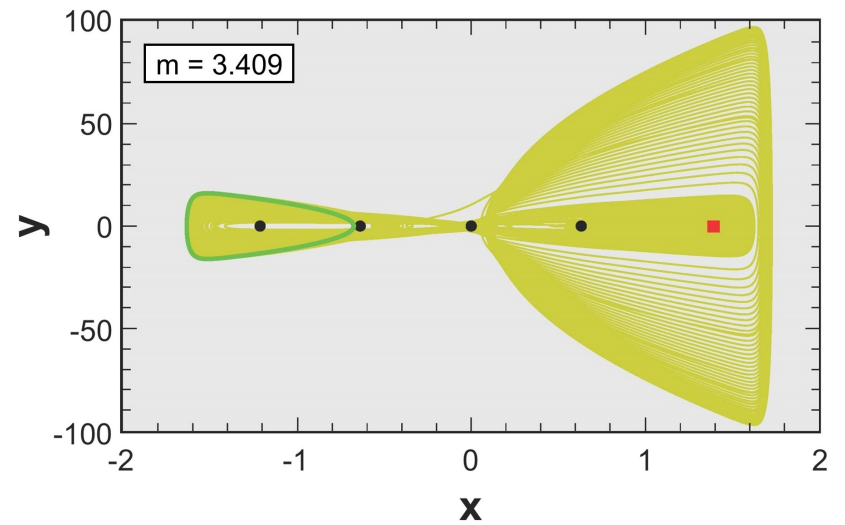

(b)

Figure 4: Metastable chaos for $m=3.409$ : (a) $x(t)$ demonstrating a transition through metastable chaos; (b) a projection of the corresponding phase trajectory onto phase plane $(x, y)$. The figure illustrates the evolution from periodic oscillations in the negative x-values (green closed curve), through metastable chaos (yellow orbit), which is winding around four unstable fixed points (black circles) until it converges to a stable fixed point at $x=1.39, y=0$ (red square). From the left to the right these fixed points correspond to ones labeled (I)-(V) in Fig. 2.

to the stable fixed point (V) (near the upper electrode).

Metastable chaos itself and transition to it are shown in Figs. 4(a) and 4(b), where $x(t)$ and the corresponding projection of the phase trajectories onto plane $(x, y)$ are presented, respectively. For $m=3.409$, the trajectory, which starts from the initial conditions on the previously disappeared limit cycle (green loop in Fig. 4(b)), first produces almost periodic oscillations of $x(t)$, whose amplitude slowly grows with time (Fig. 4(a)). At the time moment $t \approx 50$ the oscillations demonstrate an abrupt jump entering the epoch of irregular (chaotic) behaviour, which lasts for a time interval of $\Delta t \approx 300$. After this time period, the oscillations gradually

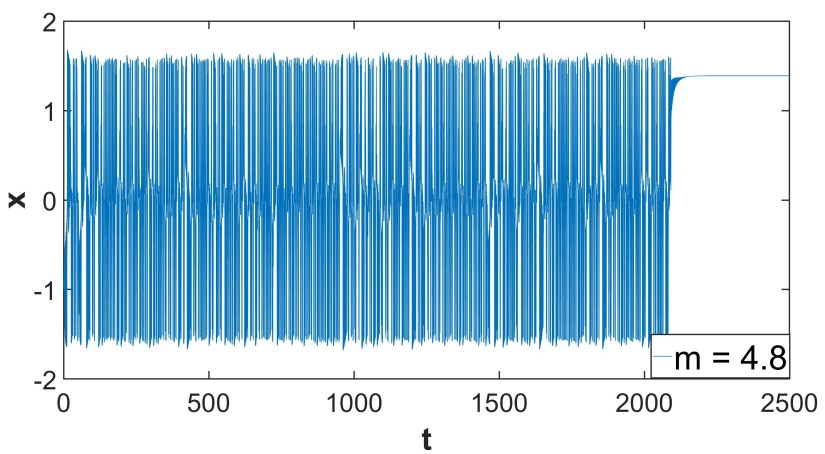

(a)

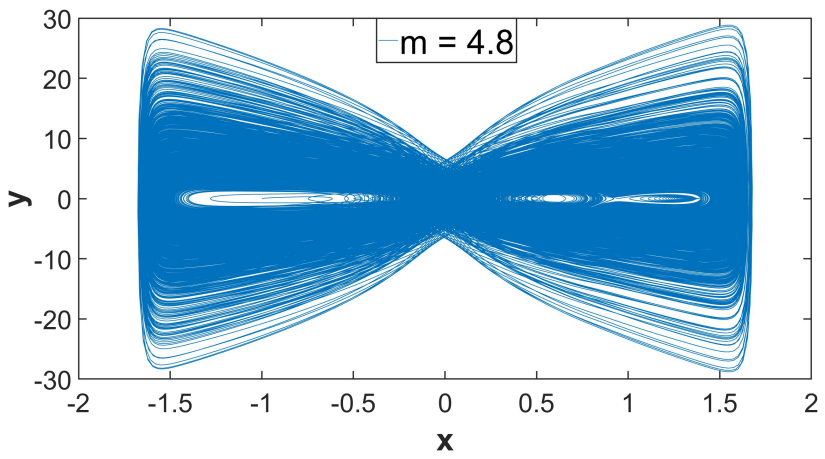

(b)

Figure 5: (a) The time dependence of nanoparticle position $x(t)$ and (b) projection of phase trajectory onto the plane $(x, y)$ for metastable chaos at mass, $m=4.8$, with initial conditions at $x(t=0)=-0.999, y(t=0)=0$.

converge to a steady state. From viewpoint of the phase space consideration (see Fig. 4(b)), the epoch of chaos is seen as a long phase trajectory (yellow curve) rambling around four unstable fixed points (black circles) until it is attracted to a stable fixed point (red square). In literature, this type of transition is referred to as metastable chaos [37] or transient chaos [38].

We believe that such transient behaviour to be analogous to metastability in neurons [18, 39], as the dynamics of our system for a long but finite time stays out of equilibrium by demonstrating rather random excursion between vicinities of unstable steady states (see e.g. [18]) finally converging to a stable fixed point. This time duration characterising the metastable chaos varies with the initial conditions and parameter values, thus has the potential to be extended or shortened as necessary. For instance, Fig. 5 demonstrates the time realization of $x(t)$ and phase trajectory plots for $m=4.8$. In this particular case chaos lasts about ten times 
longer than illustrated in Fig.4, however for some parameter values (e.g. $m=4.5$ ) the duration of metastable chaos can be even much longer and exceeds $t=10^{5}$.

\subsection{Intermittent Chaos}

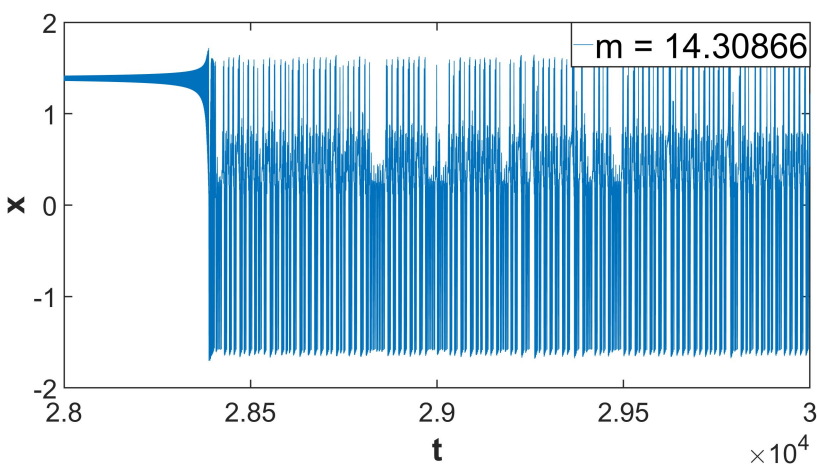

Figure 6: A plot showing how the position of particle, $x$, changes over time starting from an unstable fixed point at time, $t=0$, for mass parameter value of $m=14.30866$. The particle begins to oscillate with a very small but increasing amplitude around the fixed point until $t \approx 2.84 \times 10^{4}$, after which it enters a chaotic state.

As $m$ increases further, the stable fixed point labelled as (V) in Fig. 2 loses its stability at position $\mathrm{C}$ when $m \approx 14$ (see Fig. 2 ), which leads to onset of chaotic oscillations. Fig. 6 illustrates the transition to chaos when mass is fixed $(m=14.39866)$ and particle starts motion from the initial conditions in the unstable fixed point $(\mathrm{V})$ at $x \approx 1.39$. The nanoparticle begins to oscillate around its unstable fixed point with a very small but increasingwith-time amplitude and after a time $t \approx 2.84 \times 10^{4}$, the oscillations around $x=1.39$ reach their maximum span before becoming chaotic. A typical timerealization of chaotic oscillations of $x(t)$ and the corresponding phase trajectories for $m=14.31$ are presented in Fig. 7 (a) and (b), respectively. The oscillations switch between two distinctive modes: spikes that reach high positive amplitudes but at lower velocities (right-hand-side of the phase portrait) and sections where the oscillations are mostly in the negative $x$-values, with lower amplitudes but reaching higher velocities (left-hand-side of the phase portrait).

Such dynamical pattern is typical for intermittent chaos, and to explore this regime in more detail, we study how the oscillations evolve with variation of the applied voltage $V_{a p}$ and fixed $m=$ 14.30866. At $V_{a p}=65.868$ the oscillations are periodic. As $V_{a p}$ increases, some irregularities begin

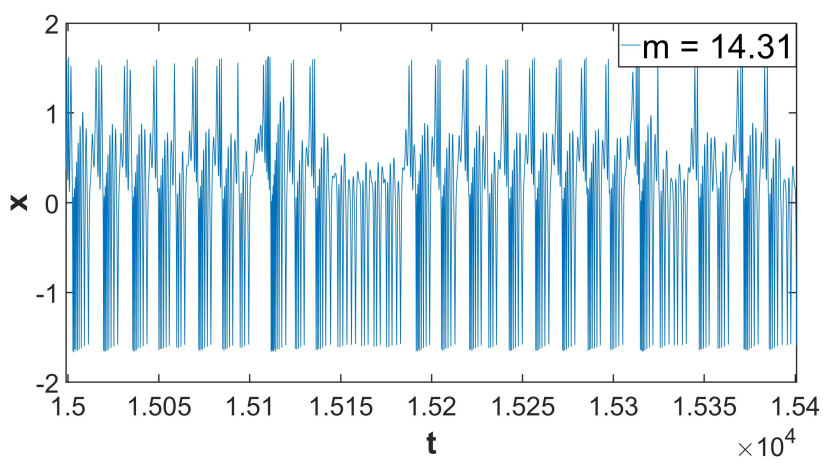

(a)

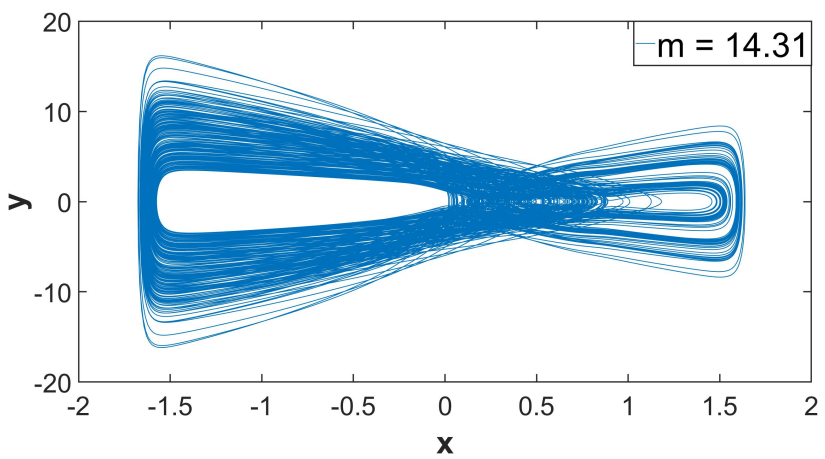

(b)

Figure 7: (a) A typical time realization of $x(t)$ and (b) the corresponding projection of the phase trajectory for $m=$ 14.31

to appear - these occur intermittently and become more common as $V_{a p}$ grows further. The plots containing the power spectral density (PSD) and a fragment of the corresponding time-realizations of $x(t)$ for different values of $V_{a p}$ shown in Fig. 8 illustrate the onset and the evolution of intermittent chaos in the system. The periodic oscillations at $V_{a p}=65.868$ have clear sharp peaks at certain frequencies in the PSD (Figure 8(a)). Figures $8(\mathrm{~b})$ and $8(\mathrm{c})$ obtained for $V_{a p}=65.869$ and $V_{a p}=70.000$, respectively, show that the PSD follows a $1 / f^{\delta}$ power law with power exponent $-\delta$. Specifically, there are two distinct values of $\delta$ - one for smaller frequencies and one for larger frequencies, which can be estimated from the slopes in the log-log graphs. The slopes of the best-fit lines have been calculated for frequencies up to $1.2 \cdot 10^{-2}$ for $V_{a p}=65.869$ and $V_{a p}=70.000$, resulting with slope values of $\delta=-0.50$ and $\delta=-0.54$, respectively. For frequencies above $\sim 1$, the slope values are -5.1 and -5.0 . The two distinct slopes for different ranges of frequency, specifically with $1 / \sqrt{(} f)$ scal- 


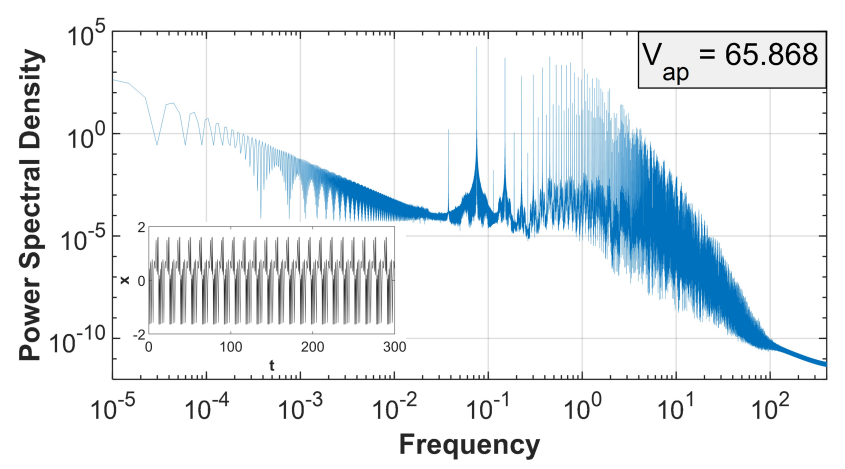

(a)

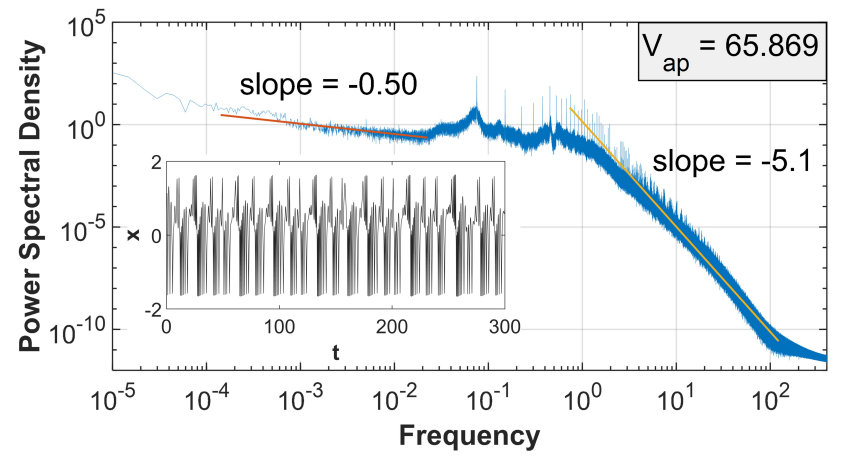

(b)

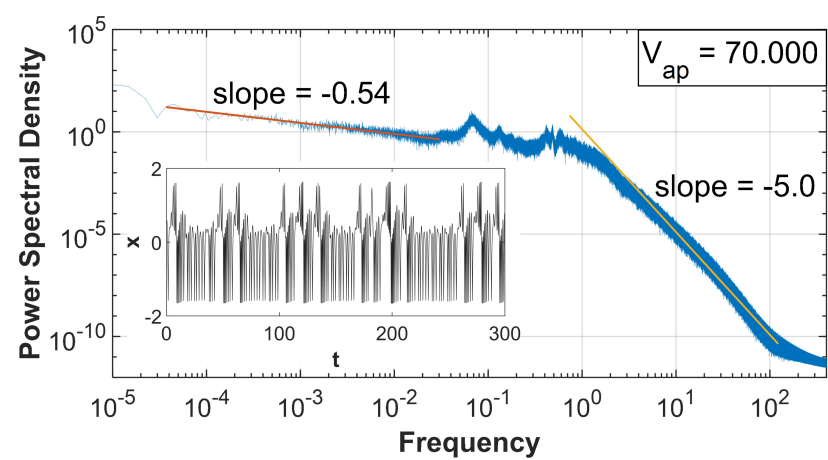

(c)

Figure 8: Power spectral density of $x(t)$ for (a) periodic oscillations at $V_{a p}=65.868$, the onset of chaotic oscillations at (b) $V_{a p}=65.869$, and the developed chaotic motion at (c) $V_{a p}=70$. The insets show the fragment of the corresponding time-realiztions of $x(t)$.

ing for lower frequencies, are characteristic of on-off intermittency [40].

In order to complete our analysis, we calculate the probability density of interspike intervals (ISIs). Oscillations of nanoparticle result in switching resistance between the high resistive state with resistance $R=R_{0} \cosh (1 / \lambda) \sim 2000 R_{0}$ and the low resistive state $R=R_{0}$ accompanied with large cur-

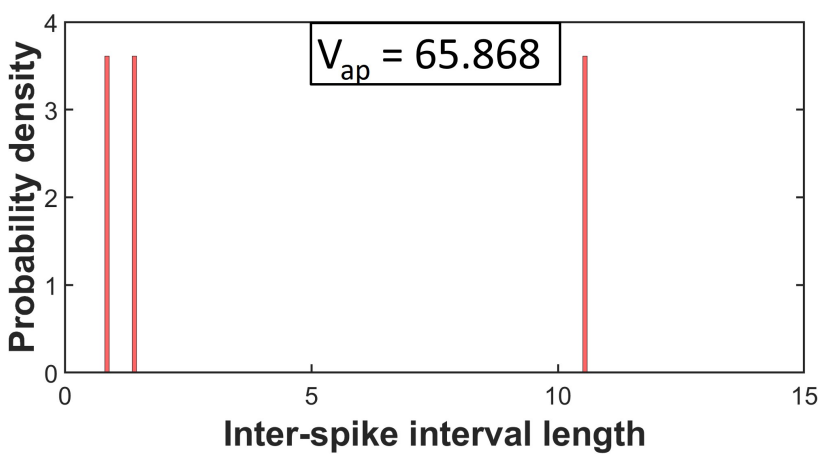

(a)

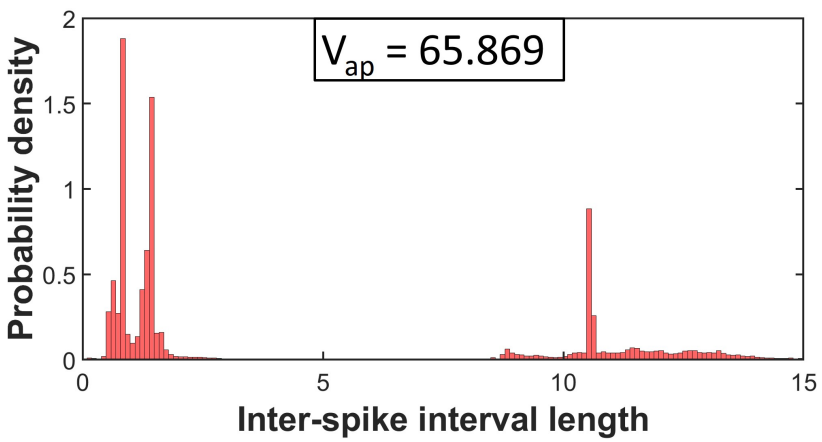

(b)

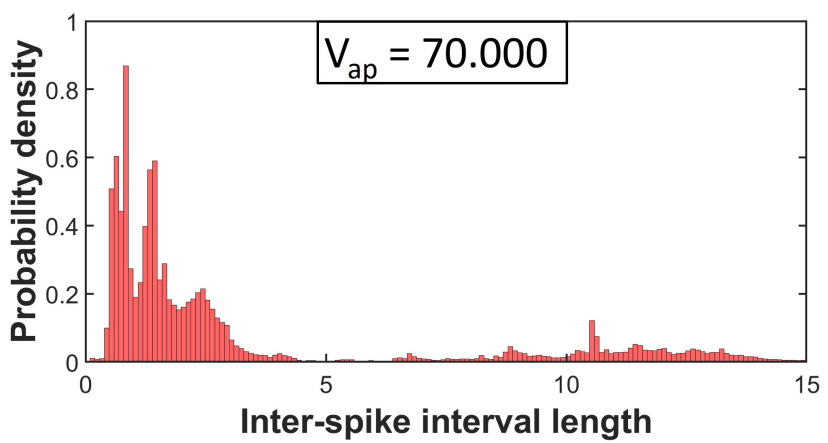

(c)

Figure 9: Probability density of interspike intervals, that is time between two successive switching of the simulated memristor to its low resistive state for (a) periodic oscillations at $V_{a p}=65.868$, the onset of chaotic oscillations at (b) $V_{a p}=65.869$ and the developed chaotic oscillations at (c) $V_{a p}=70.000$; these probability densities correspond to the spectra shown in fig. (8).

rent spikes which mimic spiking in biological neurons. For the periodic oscillations of the nanoparticle, whose spectrum is shown in Fig. 8(a), the time intervals between subsequent switchings have only three possible values, which is represented in Fig. 9 (a) by three peaks in the ISI probability density. The onset of chaos manifests itself in a slight broad- 
ening of the above peaks (Fig. 9(b)) evidencing the appearance of deviation from the periodic oscillations. In the developed chaotic regime the ISI probability distribution becomes significantly broader, but still has a fingerprint pattern with additional maxima appearing different from initial three ISI values related to periodic motion (Fig. 9(c)).

The existence of periodic and chaotic oscillations within a small parameter change makes this a promising model for artificial neurons, as it allows to match the behaviour of biological neurons, which exist at the edge of chaos.

\section{Conclusions}

We proposed a deterministic model for a diffusive artificial memristive neuron, which consists of a diffusive memristor with inertial Ag-nanoparticles, a capacitor in parallel to the memristor and a resistor. When an external DC-voltage is applied, the simulated artificial neuron generates spiking oscillations. The system demonstrates regions of periodicity, meta-stable chaos and intermittent chaos, for different values of the mass and applied voltages. Although the inertia is not easy to control in experiments, our analysis shed light on how it may transform dynamics of artificial neurons built using diffusion memristor elements. Periodic oscillations occur for values of $m$ above $m=0.26$ and below $m=3.409$ followed by metastable chaos for larger $m$. In the latter regime the neuron shows chaotic behaviour for a finite amount of time before converging to a stable fixed point. The model enters a regime of intermittent chaos at $m=14.30866$. We show that the chaotic oscillations demonstrate onoff intermittency and obey $1 / f^{\delta}$ power law, with $\delta=-1 / 2$ for lower frequencies and $\delta=-5$ for higher frequencies. Since biological neurons are known to operate at the edge of chaos, demonstrating both chaotic and non-chaotic behaviour, we believe this model has the potential to contribute to creating more bio-realistic artificial neurons.

\section{Acknowledgements}

This work was supported in part by The Engineering and Physical Sciences Research Council (EPSRC) (grant No EP/S032843/1).

\section{References}

[1] H. Weber, Big Data and Artificial Intelligence: Complete Guide to Data Science, AI, Big Data and Machine Learning. Kindle Edition, Independently published (August 12, 2020), 2020.

[2] D. E. O'Leary, Artificial intelligence and big data, IEEE intelligent systems 28 (2) (2013) 96-99.

[3] D. J. Hemanth, Artificial Intelligence Techniques for Satellite Image Analysis, Springer, 2019.

[4] Y. Guo, Y. Liu, A. Oerlemans, S. Lao, S. Wu, M. S. Lew, Deep learning for visual understanding: A review, Neurocomputing 187 (2016) 27-48.

[5] S. Belciug, Artificial Intelligence in Cancer: Diagnostic to Tailored Treatment, Academic Press, 2020.

[6] S. Huang, J. Yang, S. Fong, Q. Zhao, Artificial intelligence in cancer diagnosis and prognosis: Opportunities and challenges, Cancer Letters (2019).

[7] Y. Li, Z. Wang, R. Midya, Q. Xia, J. J. Yang, Review of memristor devices in neuromorphic computing: materials sciences and device challenges, Journal of Physics D: Applied Physics 51 (50) (2018) 503002.

[8] G. W. Burr, R. M. Shelby, A. Sebastian, S. Kim, S. Kim, S. Sidler, K. Virwani, M. Ishii, P. Narayanan, A. Fumarola, et al., Neuromorphic computing using non-volatile memory, Advances in Physics: X 2 (1) (2017) 89-124.

[9] Y. V. Pershin, S. Shevchenko, F. Nori, Memristive sisyphus circuit for clock signal generation, Scientific Reports 6 (2016) 26155 .

[10] L. Chua, Memristor-the missing circuit element, IEEE Transactions on circuit theory 18 (5) (1971) 507-519.

[11] D. B. Strukov, G. S. Snider, D. R. Stewart, R. S. Williams, The missing memristor found, Nature 453 (7191) (2008) 80-83.

[12] L. Chua, Memristor, Hodgkin-Huxley, and edge of chaos, Nanotechnology 24 (2013) 383001.

[13] S. G. Kim, J. S. Han, H. Kim, S. Y. Kim, H. W. Jang, Recent advances in memristive materials for artificial synapses, Advanced Materials Technologies 3 (12) (2018) 1800457.

[14] Z. Wang, S. Joshi, S. E. Savel'ev, H. Jiang, R. Midya, P. Lin, M. Hu, N. Ge, J. P. Strachan, Z. Li, et al., Memristors with diffusive dynamics as synaptic emulators for neuromorphic computing, Nature Materials 16 (1) (2017) 101.

[15] S. Savel'ev, F. Marchesoni, A. Bratkovsky, Mesoscopic resistive switch: non-volatility, hysteresis and negative differential resistance, European Physical Journal B 86 (2013) 501.

[16] Z. Wang, S. Joshi, S. Savel'ev, W. Song, R. Midya, Y. Li, M. Rao, P. Yan, S. Asapu, Y. Zhuo, H. Jiang, P. Lin, C. Li, J. H. Yoon, N. K. Upadhyay, J. Zhang, M. Hu, J. P. Strachan, M. Barnell, Q. Wu, H. Wu, R. S. Williams, Q. Xia, J. J. Yang, Fully memristive neural networks for pattern classification with unsupervised learning, Nature Electronics 1 (2018) 137-145.

[17] N. Bertschinger, T. Natschläger, Real-time computation at the edge of chaos in recurrent neural networks, Neural computation 16 (7) (2004) 1413-1436.

[18] M. I. Rabinovich, R. Huerta, P. Varona, V. S. Afraimovich, Transient cognitive dynamics, metastability, and decision making, PLoS computational biology 4 (5) (2008). 
[19] E. Tognoli, J. S. Kelso, The metastable brain, Neuron 81 (1) (2014) 35-48

[20] S. B. Field, M. Klaus, M. Moore, F. Nori, Chaotic dynamics of falling disks, Nature 388 (1997) 252.

[21] S. Field, N. Venturi, F. Nori, Marginal stability and chaos in coupled faults modeled by nonlinear circuits, Physical Review Letters 74 (1995) 3498.

[22] V. R. Misko, S. Savel'ev, A. L. Rakhmanov, F. Nori, Nonuniform self-organized dynamical states in superconductors with periodic pinning, Physical Review Letters 96 (2006) 127004.

[23] V. R. Misko, S. Savel'ev, A. L. Rakhmanov, F. Nori, Negative differential resistivity in superconductors with periodic arrays of pinning sites, Physical Review B 75 (2007) 024509.

[24] A. Maeda, Y. Inoue, H. Kitano, S. Savel'ev, S. Okayasu, I. Tsukada, F. Nori, Nanoscale friction: Kinetic friction of magnetic flux quanta and charge density waves, Physical Review Lettrs 94 (2005) 077001.

[25] S. Savel'ev, F. Marchesoni, F. Nori, Interacting particles on a rocked ratchet: Rectification by condensation, Physical Review E 71 (011107) (2005).

[26] M. Sciamanna, Vibrations copying optical chaos, Nature Photonics 10 (2016) 366.

[27] F. Monifi, J. Zhang, Ş. K. Özdemir, B. Peng, Y.-x. Liu, F. Bo, F. Nori, Y. L., Optomechanically induced stochastic resonance and chaos transfer between optical fields, Nature Photonics 10 (2016) 399.

[28] S. Kumar, J. P. Strachan, R. S. Williams, Chaotic dynamics in nanoscale NbO2 Mott memristors for analogue computing, Nature 548 (7667) (2017) 318.

[29] L. V. Gambuzza, L. Fortuna, M. Frasca, E. Gale, Experimental evidence of chaos from memristors, International Journal of Bifurcation and Chaos 25 (08) (2015) 1550101.

[30] R. Midya, Z. Wang, J. Zhang, S. E. Savel'ev, C. Li, M. Rao, M. H. Jang, S. Joshi, H. Jiang, P. Lin, K. Norris, N. Ge, Q. Wu, Z. Barnell, M. amd Li, H. L. Xin, R. S. Williams, Q. Xia, J. J. Yang, Anatomy of ag/hafnia-based selectors with 1010 nonlinearity, Adv. Mater. 29 (2017) 1604457.

[31] S. E. Savel'ev, A. S. Alexandrov, A. M. Bratkovsky, R. S. Williams, Molecular dynamics simulations of oxide memory resistors (memristors), Nanotechnology 22 (2011) 254011.

[32] S. E. Savel'ev, A. S. Alexandrov, A. M. Bratkovsky, R. S. Williams, Molecular dynamics simulations of oxide memristors: thermal effects, Applied Physics A 102 (2011) 891.

[33] S. E. Savel'ev, A. S. Alexandrov, A. M. Bratkovsky, R. S. Williams, Molecular dynamics simulations of oxide memristors: Crystal field effects, Applied Physics Letters 99 (2011) 053108.

[34] M. Wang, C. Bi, L. Li, S. Long, Q. Liu, H. Lv, N. Lu, P. Sun, M. Liu, Thermoelectric seebeck effect in oxidebased resistive switching memory, Nature Communications 5 (2014) 4598.

[35] S. Kumar, Z. Wang, X. Huang, N. Kumari, N. Davila, J. P. Strachan, D. Vine, A. L. D. Kilcoyne, Y. Nishi, R. S. Williams, Conduction channel formation and dissolution due to oxygen thermophoresis/diffusion in hafnium oxide memristors, ACS Nano 10 (2016) 1120511210.

[36] A. S. Alexandrov, A. M. Bratkovsky, B. Bridle, S. E. Savel'ev, D. B. Strukov, R. S. Williams, Current- controlled negative differential resistance due to joule heating in tio2, Applied Physics Letters 99 (2011) 202104.

[37] J. A. Yorke, E. D. Yorke, Metastable chaos: The transition to sustained chaotic behavior in the Lorenz model, Journal of Statistical Physics 21 (3) (1979) 263-277.

[38] T. Lilienkamp, U. Parlitz, Terminal transient phase of chaotic transients, Physical Review Letters 120 (9) (2018) 094101

[39] J. A. Roberts, L. L. Gollo, R. G. Abeysuriya, G. Roberts, P. B. Mitchell, M. W. Woolrich, M. Breakspear, Metastable brain waves, Nature Communications 10 (1) (2019) 1056.

[40] T. John, U. Behn, R. Stannarius, Fundamental scaling laws of on-off intermittency in a stochastically driven dissipative pattern-forming system, Physical Review E 65 (4) (2002) 046229. 